\title{
EXTENDED FIBONACCI NUMBERS AND POLYNOMIALS WITH PROBABILITY APPLICATIONS
}

\author{
DEMETRIOS L. ANTZOULAKOS
}

Received 30 April 2004

\begin{abstract}
The extended Fibonacci sequence of numbers and polynomials is introduced and studied. The generating function, recurrence relations, an expansion in terms of multinomial coefficients, and several properties of the extended Fibonacci numbers and polynomials are obtained. Interesting relations between them and probability problems which take into account lengths of success and failure runs are also established.
\end{abstract}

2000 Mathematics Subject Classification: 62E15, 11B39, 60 C05.

1. Introduction. Let $F_{n}^{(k)}, n \geq 0$, be the sequence of Fibonacci numbers of order $k$. It is well known that $F_{n+2}^{(k)}$ enumerates the number of different ways in which $n$ (in total) symbols $\{0,1\}$ can be arranged in a line in such a way that $k$ (or more) consecutive 1's do not appear. Philippou and Muwafi [12] showed that the number of arrangements of $\{0,1\}$-sequences of length $n$, such that the last is 0 and $k$ consecutive 1 's do not appear, is given by $F_{n+1}^{(k)}$. Also, they showed that the probability mass function (pmf) of the random variable $N_{k}$, which denotes the number of $\{0,1\}$ Bernoulli trials until the occurrence of the $k$ th consecutive success (1 denotes success), is given by $\operatorname{Pr}\left(N_{k}=\right.$ $n+k)=F_{n+1}^{(k)} / 2^{n+k}, n \geq 0$, in the symmetric case, that is, when the probability $p$ of success is equal to $1 / 2$.

The distribution of the random variable $N_{k}$ has been termed as geometric distribution of order $k$ by Philippou et al. [9] who derived from its study the negative binomial and the Poisson distribution of order $k$. Since then, an upsurge of interest has been observed in the distributions of success/failure runs, as the extensive recent literature indicates (for a recent review, see, e.g., Balakrishnan and Koutras [4]).

Motivated by the work of Philippou and Muwafi [12] and their work in [9], Philippou et al. [8] introduced and studied the sequence of Fibonacci polynomials of order $k$ and expressed the pmf of the random variable $N_{k}$ in terms of these polynomials. Philippou et al. [10] introduced and studied a more flexible sequence of polynomials, the sequence of Fibonacci-type polynomials of order $k$, and denoted it by $F_{n}^{(k)}(x), n \geq 0$. The sequence of Fibonacci-type polynomials of order $k$ is defined by $F_{0}^{(k)}(x)=0, F_{1}^{(k)}(x)=1$, and the recurrence relation

$$
F_{n}^{(k)}(x)= \begin{cases}x \sum_{i=1}^{n} F_{n-i}^{(k)}(x), & 2 \leq n \leq k+1, \\ x \sum_{i=1}^{k} F_{n-i}^{(k)}(x), & n \geq k+2 .\end{cases}
$$


This sequence of polynomials includes as a special case the Fibonacci sequence of order $k$, since $F_{n}^{(k)}(1)=F_{n}^{(k)}, n \geq 0$.

We recall the following results from Philippou et al. [10] and Philippou and Makri [11]:

$$
F_{n}^{(k)}(x)=(1+x) F_{n-1}^{(k)}(x)-x F_{n-k-1}^{(k)}(x), \quad n \geq 3,
$$

along with the convention that $F_{n}^{(k)}(x)=0$ for $n<0$,

$$
\begin{gathered}
G_{k}(x ; z)=\sum_{n=0}^{\infty} F_{n+1}^{(k)}(x) z^{n}=\frac{1}{1-x\left(z+z^{2}+\cdots+z^{k}\right)}=\frac{1-z}{1-(1+x) z+x z^{k+1}}, \\
\operatorname{Pr}\left(N_{k}=n+k\right)=p^{n+k} F_{n+1}^{(k)}\left(\frac{q}{p}\right), \quad n \geq 0, \\
\operatorname{Pr}\left(L_{n}<k\right)=\frac{p^{n+1}}{q} F_{n+2}^{(k)}\left(\frac{q}{p}\right), \quad 0 \leq n \leq k-1,
\end{gathered}
$$

(in (1.5), $L_{n}$ denotes the length of the longest success run in $n$ Bernoulli trials).

In all of the above results the interest focuses exclusively on runs of 1 's. In the present paper, we consider $\{0,1\}$-sequences of length $n$, where both runs of 1 's and 0 's are taken into account. In Section 2, we introduce and study the sequence of extended Fibonacci numbers, denoted by $A_{n}^{(k, r)}(n \geq 0, k, r \geq 2)$. The $n$th term of this sequence is defined to be the number of arrangements of $\{0,1\}$-sequences of length $n$ such that the last is 0 and there do not exist any $k$ consecutive 1's (or more) and any $r$ consecutive 0's (or more). It is obvious that $A_{n}^{(k, r)}=F_{n+1}^{(k)}$ for $n<r$, or equivalently, $\lim _{r \rightarrow \infty} A_{n}^{(k, r)}=F_{n+1}^{(k)}$, $n \geq 0$. The generating function, recurrence relations, an expansion in terms of multinomial coefficients, and several interesting properties of this sequence are established. In Section 3, we introduce and study the sequence of extended Fibonacci polynomials, denoted by $A_{n}^{(k, r)}(x)(n \geq 0, k, r \geq 2)$, and analogous results are obtained. This sequence constitutes an appropriate generalization of the numbers $A_{n}^{(k, r)}$ in the sense that $A_{n}^{(k, r)}(1)=A_{n}^{(k, r)}, n \geq 0$.

In Section 4, we consider the random variable $W$ which denotes the number of Bernoulli trials with common success probability $p$ until the occurrence of the $k$ th consecutive success or the $r$ th consecutive failure, whichever comes sooner. The random variable $W$ has been initially studied by Feller [6, page 327] (see also Ebneshahrashoob and Sobel [5]), and it is closely related to problems of practical interest in statistical theory of runs and reliability theory (see, e.g., Koutras [7], Aki et al. [1], and the references therein). The pmf of $W$ is expressed directly in terms of the polynomials $A_{n}^{(k, r)}(x)$ offering an efficient way for the computation of the pmf of $W$, which is simpler from the respective ways presented by Antzoulakos and Philippou [2, 3]. Relations between the polynomials $A_{n}^{(k, r)}(x)$ and probability problems which take into account lengths of success and failure runs in a fixed number of $n$ Bernoulli trials are also established.

In order to avoid unnecessary repetitions, we mention here that whenever sums and products are taken over $i$ and $j$, ranging from 1 to $k-1$ and from 1 to $r-1$, respectively, 
we will omit these limits for notational simplicity. Furthermore, whenever sums of the form $\sum_{m=a}^{b} g(m)$ with $a>b$ appear, they are taken to be zero.

2. Sequence of extended Fibonacci numbers. In the present section, we introduce and study a new sequence of numbers which will be denoted by $A_{n}^{(k, r)}(n \geq 0, k, r \geq 2)$. The $n$th term of this sequence is defined to be the number of arrangements of $\{0,1\}$ sequences of length $n$ such that the last is 0 and there do not exist any $k$ consecutive 1 's (or more) and any $r$ consecutive 0's (or more). It follows from the above definition that $A_{n}^{(k, r)}=F_{n+1}^{(k)}$ for $n<r$, or equivalently, $\lim _{r \rightarrow \infty} A_{n}^{(k, r)}=F_{n+1}^{(k)}, n \geq 0$. We call this sequence the sequence of extended Fibonacci numbers. In the following two theorems we derive an expansion of $A_{n}^{(k, r)}$ in terms of multinomial coefficients and the generating function of the numbers $A_{n}^{(k, r)}$, respectively.

THEOREM 2.1. Let $A_{n}^{(k, r)}, n \geq 0$, be the sequence of the extended Fibonacci numbers. With the convention $A_{0}^{(k, r)}=1$,

$$
A_{n}^{(k, r)}=\sum_{m=0}^{r-1} \sum_{\sum_{i} \sum_{j}(i+j) n_{i j}=n-m} \frac{\left(\sum_{i} \sum_{j} n_{i j}\right) !}{\prod_{i} \prod_{j} n_{i j} !}, \quad n \geq 0 .
$$

Proof. First we observe that (2.1) is true for $n=0$. Assume now that $n \geq 1$. Modifying a combinatorial argument of Philippou and Muwafi [12] (see also Antzoulakos and Philippou [2]), we observe that an arrangement of $\{0,1\}$-sequences of length $n$ belongs to the ones enumerated by $A_{n}^{(k, r)}$ if and only if it is of the form

$$
\underbrace{00 \cdots 0}_{m} E_{1} E_{2} \cdots E_{\Sigma_{i} \Sigma_{j} n_{i j}}, \quad 0 \leq m \leq r-1,1 \leq i \leq k-1,1 \leq j \leq r-1
$$

such that $n_{i j}$ of the $E^{\prime}$ s are of the form $\underbrace{11 \cdots 1}_{i} \underbrace{00 \cdots 0}_{j}$, where $1 \leq i \leq k-1$ and $1 \leq j \leq$ $r-1$, and the nonnegative integers $n_{i j}$ satisfy the condition $\sum_{i} \sum_{j}(i+j) n_{i j}=n-m$. Fix $m$ and $n_{i j}$. Then the number of the above arrangements is

$$
\frac{\left(\sum_{i} \sum_{j} n_{i j}\right) !}{\prod_{i} \prod_{j} n_{i j} !}
$$

The proof of the theorem then follows easily by noting that $m$ and $n_{i j}$ may vary subject to the conditions $0 \leq m \leq r-1$ and $\sum_{i} \sum_{j}(i+j) n_{i j}=n-m$.

THEOREM 2.2. Let $A_{k, r}(z)$ be the generating function of the sequence of the extended Fibonacci numbers $A_{n}^{(k, r)}, n \geq 0$. Then

$$
A_{k, r}(z)=\frac{1+z+z^{2}+\cdots+z^{r-1}}{1-\left(z+z^{2}+\cdots+z^{r-1}\right)\left(z+z^{2}+\cdots+z^{k-1}\right)} .
$$


Proof. Using Theorem 2.1, we get

$$
\begin{aligned}
A_{k, r}(z) & =\sum_{n=0}^{\infty} A_{n}^{(k, r)} z^{n} \\
& =\sum_{n=0}^{\infty} \sum_{m=0}^{r-1} z^{m} \sum_{\sum_{i} \sum_{j}(i+j) n_{i j}=n-m} \frac{\left(\sum_{i} \sum_{j} n_{i j}\right) !}{\prod_{i} \prod_{j} n_{i j} !} \prod_{i} \prod_{j}\left(z^{i+j}\right)^{n_{i j}} \\
& =\sum_{m=0}^{r-1} z^{m} \sum_{S_{m, n}} \frac{\left(\sum_{i} \sum_{j} n_{i j}\right) !}{\prod_{i} \prod_{j} n_{i j} !} \prod_{i} \prod_{j}\left(z^{i+j}\right)^{n_{i j}},
\end{aligned}
$$

where $S_{m, n}(0 \leq m \leq r-1)$ denotes the set of nonnegative solutions of the equation $\sum_{i} \sum_{j}(i+j) n_{i j}=n-m$ for $0 \leq n<\infty$. We observe that $S_{m, n}=S_{n}$, where $S_{n}$ denotes the set of nonnegative solutions of the equation $\sum_{i} \sum_{j} n_{i j}=n$ for $0 \leq n<\infty$. Thus, utilizing the multinomial theorem, we get

$$
\begin{aligned}
A_{k, r}(z) & =\left(\sum_{m=0}^{r-1} z^{m}\right) \sum_{S_{n}} \frac{\left(\sum_{i} \sum_{j} n_{i j}\right) !}{\prod_{i} \prod_{j} n_{i j} !} \prod_{i} \prod_{j}\left(z^{i+j}\right)^{n_{i j}} \\
& =\left(\sum_{m=0}^{r-1} z^{m}\right) \sum_{n=0}^{\infty} \sum_{\sum_{i} \sum_{j} n_{i j}=n} \frac{\left(\sum_{i} \sum_{j} n_{i j}\right) !}{\prod_{i} \prod_{j} n_{i j} !} \prod_{i} \prod_{j}\left(z^{i+j}\right)^{n_{i j}} \\
& =\left(\sum_{m=0}^{r-1} z^{m}\right) \sum_{n=0}^{\infty}\left(\sum_{i} \sum_{j} z^{i+j}\right)^{n} \\
& =\left(\sum_{m=0}^{r-1} z^{m}\right)\left(1-\sum_{i} z^{i} \sum_{j} z^{j}\right)^{-1}
\end{aligned}
$$

which completes the proof of the theorem.

The evaluation of the sequence $A_{n}^{(k, r)}$ via Theorem 2.1 is not a simple task since it involves solutions of Diophantine equations. In the following proposition, we derive a simple recursive scheme for the evaluation of the sequence $A_{n}^{(k, r)}$.

Proposition 2.3. The sequence of the extended Fibonacci numbers $A_{n}^{(k, r)}$ satisfies the following recursive scheme:

$$
A_{n}^{(k, r)}= \begin{cases}1, & n=0,1, \\ 1+\sum_{\substack{i \\ i+j \leq n}} A_{n-(i+j)}^{(k, r)}, & 2 \leq n \leq r-1, \\ \sum_{\substack{i \\ i+j \leq n}} A_{n-(i+j)}^{(k, r)}, & r \leq n \leq k+r-2, \\ \sum_{i} \sum_{j} A_{n-(i+j)}^{(k, r)}, & n \geq k+r-1,\end{cases}
$$

with the convention that $A_{n}^{(k, r)}=0$ for $n<0$. 
Proof. Theorem 2.2 implies that

$$
\sum_{n=0}^{\infty} A_{n}^{(k, r)} z^{n}\left(1-\sum_{i} \sum_{j} z^{i+j}\right)=1+z+z^{2}+\cdots+z^{r-1}
$$

which may be written equivalently as

$$
\sum_{n=0}^{\infty} A_{n}^{(k, r)} z^{n}-\sum_{i} \sum_{j} \sum_{n=0}^{\infty} A_{n-(i+j)}^{(k, r)} z^{n}=1+z+z^{2}+\cdots+z^{r-1} .
$$

The proof of Proposition 2.3 follows by equating the coefficients of $z^{n}$ on both sides of the above equation.

A more efficient recursive scheme satisfied by the sequence of the extended Fibonacci numbers is presented in the following proposition.

Proposition 2.4. The sequence of the extended Fibonacci numbers $A_{n}^{(k, r)}$ satisfies the following recursive scheme:

$$
A_{n}^{(k, r)}= \begin{cases}1, & n=0,1, \\ 2 A_{r-1}^{(k, r)}-A_{r-k-1}^{(k, r)}-1, & n=r, \\ 2 A_{r}^{(k, r)}-A_{r-k}^{(k, r)}, & n=r+1, \\ 2 A_{n-1}^{(k, r)}-A_{n-r-1}^{(k, r)}-A_{n-k-1}^{(k, r)}+A_{n-k-r}^{(k, r)}, & n \geq 2, n \neq r, r+1,\end{cases}
$$

with the convention that $A_{n}^{(k, r)}=0$ for $n<0$.

Proof. It follows from Theorem 2.2 that the generating function $A_{k, r}(z)$ of the sequence $A_{n}^{(k, r)}, n \geq 0$, may be written as

$$
A_{k, r}(z)=\frac{1-z-z^{r}+z^{r+1}}{1-2 z+z^{r+1}+z^{k+1}-z^{k+r}}
$$

which implies that

$$
\sum_{n=0}^{\infty}\left(A_{n}^{(k, r)}-2 A_{n}^{(k, r)} z+A_{n}^{(k, r)} z^{r+1}+A_{n}^{(k, r)} z^{k+1}-A_{n}^{(k, r)} z^{k+r}\right) z^{n}=1-z-z^{r}+z^{r+1} .
$$

Equating the coefficients of $z^{n}$ on both sides of the above equation, we may easily complete the proof of Proposition 2.4.

The sequence of the extended Fibonacci numbers $A_{n}^{(k, r)}$ has a number of interesting properties. In the sequel we report a few of them.

(P1) $A_{n}^{(k, r)}=F_{n+1}^{(k)}$ for $0 \leq n<r$, or equivalently, $\lim _{r \rightarrow \infty} A_{n}^{(k, r)}=F_{n+1}^{(k)}, n \geq 0 .((\mathrm{P} 1)$ follows by the fact that when $1 \leq n<r$, then the number $A_{n}^{(k, r)}$ reduces to the number of arrangements of $\{0,1\}$-sequences of length $n$ which end with 0 and $k$ consecutive 1 's do not appear.) 
(P2) $A_{n}^{(k+1, k+1)}=F_{n+1}^{(k)}$ for $n \geq 0$. ((P2) follows by the fact that $A_{k+1, k+1}(z)=G_{k}(1 ; z)$ which may be easily derived using (1.2) for $x=1$ and Theorem 2.2. (P2) leads to a new and very interesting interpretation of the number $F_{n+1}^{(k)}$ via the interpretation of the number $A_{n}^{(k+1, k+1)}$.)

(P3) $A_{n}^{(r, k)}$ denotes the number of arrangements of $\{0,1\}$-sequences of length $n$ which end with 1 , such that in each arrangement there do not exist any $k$ consecutive 1's (or more) and any $r$ consecutive 0 's (or more). ((P3) may be easily verified by Theorem 2.1 or by symmetry reasons.)

(P4) The number, say $B_{n}^{(k, r)}$, of arrangements of $\{0,1\}$-sequences of length $n(\geq 1)$, such that in each arrangement there do not exist any $k$ consecutive 1's (or more) and any $r$ consecutive 0's (or more), is given by $B_{n}^{(k, r)}=A_{n}^{(k, r)}+A_{n}^{(r, k)}$. ((P4) follows directly from the definition of the number $A_{n}^{(k, r)}$ and (P3).)

(P5) The number of arrangements of $\{0,1\}$-sequences of length $n(\geq 1)$, which have either a run of 1's of length at least $k$ or a run of 0 's of length at least $r$, is given by $2^{n}-B_{n}^{(k, r)}$. ((P5) follows immediately from (P4).)

(P6) The number of arrangements of $\{0,1\}$-sequences of length $n(\geq k-1)$, in which the length of the longest run of 1's is $k-1$ and there are no $r$ consecutive 0 's, is given by $B_{n}^{(k, r)}-B_{n}^{(k-1, r)}$. ((P6) follows immediately from (P4).)

3. Sequence of extended Fibonacci polynomials. Presently, we introduce and study a new sequence of polynomials, denoted by $A_{n}^{(k, r)}(x)(n \geq 0, k, r \geq 2)$. The polynomials $A_{n}^{(k, r)}(x)$ constitute an appropriate extension of the numbers $A_{n}^{(k, r)}$ in the sense that $A_{n}^{(k, r)}(1)=A_{n}^{(k, r)}, n \geq 0$. We call this sequence the sequence of extended Fibonacci polynomials.

We begin with the definition of the sequence of extended Fibonacci polynomials $A_{n}^{(k, r)}(x)$, and subsequently we derive its generating function.

DEFINITION 3.1. The sequence of the extended Fibonacci polynomials $A_{n}^{(k, r)}(x)$ is given by

$$
A_{n}^{(k, r)}(x)= \begin{cases}1, & n=0, \\ x, & n=1, \\ x^{n}+\sum_{\substack{i \\ i+j \leq n}} x^{j} A_{n-(i+j)}^{(k, r)}, & 2 \leq n \leq r-1, \\ \sum_{i} \sum_{j} x^{j} A_{n-(i+j)}^{(k, r)}, & r \leq n \leq k+r-2, \\ i+j \leq n \\ \sum_{i} \sum_{j} x^{j} A_{n-(i+j)}^{(k, r)}, & n \geq k+r-1,\end{cases}
$$

with the convention that $A_{n}^{(k, r)}(x)=0$ for $n<0$. 
THEOREM 3.2. Let $A_{k, r}(x ; z)$ be the generating function of the extended Fibonacci polynomials $A_{n}^{(k, r)}(x)$. Then

$$
A_{k, r}(x ; z)=\frac{1+x z+(x z)^{2}+\cdots+(x z)^{r-1}}{1-\left(x z+(x z)^{2}+\cdots+(x z)^{r-1}\right)\left(z+z^{2}+\cdots+z^{k-1}\right)} .
$$

Proof. Using Definition 3.1, we get

$$
\begin{aligned}
A_{k, r}(x ; z)= & +x z+\sum_{n=2}^{r-1}\left(x^{n}+\sum_{\substack{i \\
i+j \leq n}} \sum_{j}^{j} A_{n-(i+j)}^{(k, r)}\right) z^{n} \\
& +\sum_{n=r}^{k+r-2} \sum_{i} \sum_{j} x^{j} A_{n-(i+j)}^{(k, r)} z^{n}+\sum_{n=k+r-1}^{\infty} \sum_{i} \sum_{j} x^{j} A_{n-(i+j)}^{(k, r)} z^{n} \\
= & \sum_{m=0}^{r-1}(x z)^{m}+\sum_{i} \sum_{j} z^{i}(z x)^{j} \sum_{n=i+j}^{r-1} A_{n-(i+j)}^{(k, r)} z^{n-(i+j)} \\
& +\sum_{i} \sum_{j} z^{i}(z x)^{j} \sum_{n=r}^{k+r-2} A_{n-(i+j)}^{(k, r)} z^{n-(i+j)} \\
& +\sum_{i} \sum_{j} z^{i}(z x)^{j} \sum_{n=k+r-1}^{\infty} A_{n-(i+j)}^{(k, r)} z^{n-(i+j)} \\
= & \sum_{m=0}^{r-1}(x z)^{m}+\sum_{i} \sum_{j} z^{i}(z x)^{j} \sum_{n=i+j}^{\infty} A_{n-(i+j)}^{(k, r)} z^{n-(i+j)} \\
= & \sum_{m=0}^{r-1}(x z)^{m}+\sum_{i} \sum_{j} z^{i}(z x)^{j} A_{k, r}(x ; z) .
\end{aligned}
$$

The proof of Theorem 3.2 follows by solving the last equation for $A_{k, r}(x ; z)$.

In the following two propositions, we present a closed formula in terms of multinomial coefficients for $A_{n}^{(k, r)}(x)$ and a recursive scheme alternative to Definition 3.1, which is satisfied by $A_{n}^{(k, r)}(x)$, respectively.

Proposition 3.3. Let $A_{n}^{(k, r)}(x), n \geq 0$, be the sequence of the extended Fibonacci polynomials. Then

$$
A_{n}^{(k, r)}(x)=\sum_{m=0}^{r-1} x^{m} \sum_{\sum_{i} \sum_{j}(i+j) n_{i j}=n-m} \frac{\left(\sum_{i} \sum_{j} n_{i j}\right) !}{\prod_{i} \prod_{j} n_{i j} !} \prod_{i} \prod_{j}\left(x^{j}\right)^{n_{i j}} .
$$

Proof. The proof of the proposition may be easily established using Theorem 3.2 and the proof of Theorem 2.2 in reverse order. 
Proposition 3.4. The sequence of the extended Fibonacci polynomials $A_{n}^{(k, r)}(x)$ satisfies the following recursive scheme:

$$
A_{n}^{(k, r)}(x)= \begin{cases}1, & n=0, \\ x, & n=1, \\ (1+x) A_{r-1}^{(k, r)}(x)-x A_{r-k-1}^{(k, r)}(x)-x^{r}, & n=r, \\ (1+x) A_{r}^{(k, r)}(x)-x A_{r-k}^{(k, r)}(x), & n=r+1, \\ (1+x) A_{n-1}^{(k, r)}(x)-x^{r} A_{n-r-1}^{(k, r)}(x) & \\ -x A_{n-k-1}^{(k, r)}(x)+x^{r} A_{n-k-r}^{(k, r)}(x), & n \geq 2, n \neq r, r+1,\end{cases}
$$

with the convention that $A_{n}^{(k, r)}(x)=0$ for $n<0$.

Proof. It follows from Theorem 3.2 that

$$
A_{k, r}(x ; z)=\frac{1-z-x^{r} z^{r}+x^{r} z^{r+1}}{1-(1+x) z+x^{r} z^{r+1}+x z^{k+1}-x^{r} z^{k+r}} .
$$

Therefore

$$
\begin{gathered}
1-z-x^{r} z^{r}+x^{r} z^{r+1} \\
=\sum_{n=0}^{\infty}\left\{A_{n}^{(k, r)}(x)-(1+x) z A_{n}^{(k, r)}(x)+x^{r} z^{r+1} A_{n}^{(k, r)}(x)\right. \\
\left.\quad+x z^{k+1} A_{n}^{(k, r)}(x)-x^{r} z^{k+r} A_{n}^{(k, r)}(x)\right\} z^{n} .
\end{gathered}
$$

Equating the coefficients of $z^{n}$ on both sides of the above equation, we may easily complete the proof of Proposition 3.4.

Next, we give an interesting identity which relates the polynomial $A_{n}^{(r, k)}(x / y)$ with the polynomial $A_{n}^{(k, r)}(y / x)$.

Proposition 3.5. Let $A_{n}^{(k, r)}(x)$ be the sequence of the extended Fibonacci polynomials. Then

$$
y^{n} A_{n}^{(r, k)}\left(\frac{x}{y}\right)=x^{n} \sum_{i=1}^{k-1} A_{n-i}^{(k, r)}\left(\frac{y}{x}\right), \quad n \geq 1 .
$$

Proof. Using Theorem 3.2, we obtain

$$
\begin{aligned}
\sum_{n=1}^{\infty} y^{n} A_{n}^{(r, k)}\left(\frac{x}{y}\right) z^{n} \\
=A_{r, k}\left(\frac{x}{y} ; y z\right)-1 \\
=\frac{\left(1+y z+(y z)^{2}+\cdots+(y z)^{r-1}\right)\left(x z+(x z)^{2}+\cdots+(x z)^{k-1}\right)}{1-\left(x z+(x z)^{2}+\cdots+(x z)^{k-1}\right)\left(y z+(y z)^{2}+\cdots+(y z)^{r-1}\right)} \\
=\left(x z+(x z)^{2}+\cdots+(x z)^{k-1}\right) \sum_{n=0}^{\infty} A_{n}^{(k, r)}\left(\frac{y}{x}\right)(x z)^{n} .
\end{aligned}
$$


The proof of Proposition 3.5 follows by equating the coefficients of $z^{n}$ on both sides of the above equation.

In ending this section, we mention a few properties of the sequence of polynomials $A_{n}^{(k, r)}(x)$ :

$$
\begin{gathered}
A_{n}^{(k, r)}(1)=A_{n}^{(k, r)}, \quad n \geq 0, \\
A_{n}^{(k, r)}(x)=F_{n+1}^{(k)}(x), \quad 0 \leq n<r, \\
\lim _{r \rightarrow \infty} A_{n}^{(k, r)}(x)=F_{n+1}^{(k)}(x) .
\end{gathered}
$$

Relation (3.10) is obvious, while relation (3.11) may be verified by relation (1.2) and Proposition 3.4, which implies that

$$
A_{n}^{(k, r)}(x)=(1+x) A_{n-1}^{(k, r)}(x)-x A_{n-k-1}^{(k, r)}(x), \quad 2 \leq n<r .
$$

Relation (3.12) is equivalent to relation (3.11) and follows by the fact that $\lim _{r \rightarrow \infty} A_{k, r}(x$; $z)=G_{k}(x ; z)$ (see also relation $(1.3)$ ).

Furthermore, we mention that relation (3.10) and Proposition 3.5 for $x=y=1$ imply that

$$
A_{n}^{(r, k)}=\sum_{i=1}^{k-1} A_{n-i}^{(k, r)}, \quad n \geq 1,
$$

and therefore the numbers $B_{n}^{(k, r)}$, introduced in (P4) of Section 2, may be expressed in the alternative form

$$
B_{n}^{(k, r)}=\sum_{i=0}^{k-1} A_{n-i}^{(k, r)}, \quad n \geq 1
$$

4. Probability applications. Let $\left\{X_{n}, n=1,2, \ldots\right\}$ be a sequence of independent Bernoulli trials with common success probability $p(0<p<1)$, and let $W$ be a random variable denoting the waiting time until a success run of length $k$ (event $E_{1}$ ) or a failure run of length $r$ (event $E_{0}$ ) occurs, whichever comes sooner. Denote by $P(n)$ the pmf of the random variable $W$, that is, $P(n)=\operatorname{Pr}(W=n)$. Also, let $P_{1}(n)\left(P_{0}(n)\right)$ be the probability that at the $n$th trial the sooner event between $E_{1}$ and $E_{0}$ occurs and the sooner event is $E_{1}\left(E_{0}\right)$. Feller [6, page 327] (see also Ebneshahrashoob and Sobel [5] and Antzoulakos and Philippou [2]) derived the probability generating function of the random variable $W$ which is given by

$$
H(z)=\sum_{n=0}^{\infty} P(n) z^{n}=\sum_{n=0}^{\infty} P_{1}(n) z^{n}+\sum_{n=0}^{\infty} P_{0}(n) z^{n}=H_{1}(z)+H_{0}(z),
$$


where

$$
\begin{aligned}
& H_{1}(z)=(p z)^{k} \frac{(1-p z)\left[1-(q z)^{r}\right]}{(1-p z)(1-q z)-(p z)(q z)\left[1-(p z)^{k-1}\right]\left[1-(q z)^{r-1}\right]}, \\
& H_{0}(z)=(q z)^{r} \frac{(1-q z)\left[1-(p z)^{k}\right]}{(1-p z)(1-q z)-(p z)(q z)\left[1-(p z)^{k-1}\right]\left[1-(q z)^{r-1}\right]} .
\end{aligned}
$$

In the present section, we relate the pmf of the random variable $W$ and probabilities which are related with lengths of success and failure runs in terms of the polynomials $A_{n}^{(k, r)}(x), n \geq 0$.

THEOREM 4.1. Let $A_{n}^{(k, r)}(x)$ be the sequence of the extended Fibonacci polynomials. Then the $\operatorname{pmf} P(n), n \geq 0$, of the random variable $W$ is given by

$$
P(n)=p^{n} A_{n-k}^{(k, r)}\left(\frac{q}{p}\right)+q^{n} A_{n-r}^{(r, k)}\left(\frac{p}{q}\right) .
$$

Proof. Relation (4.2) and Theorem 3.2 imply that

$$
\begin{aligned}
H_{1}(z) & =\sum_{n=0}^{\infty} P_{1}(n) z^{n} \\
& =(p z)^{k} \frac{1+q z+(q z)^{2}+\cdots+(q z)^{r-1}}{1-\left[p z+(p z)^{2}+\cdots+(p z)^{k-1}\right]\left[q z+(q z)^{2}+\cdots+(q z)^{r-1}\right]} \\
& =(p z)^{k} A_{k, r}\left(\frac{q}{p} ; p z\right)
\end{aligned}
$$

from which we get that

$$
P_{1}(n)=p^{n} A_{n-k}^{(k, r)}\left(\frac{q}{p}\right), \quad n \geq 0 .
$$

In a similar way, using relation (4.3) and Theorem 3.2, we may derive that

$$
P_{0}(n)=q^{n} A_{n-r}^{(r, k)}\left(\frac{p}{q}\right), \quad n \geq 0 .
$$

The proof of Theorem 4.1 then follows by relations (4.1), (4.6), and (4.7).

Theorem 4.1 and Proposition 3.4 offer an efficient way for the computation of the pmf of $W$, which is simpler from the respective ways presented by Antzoulakos and Philippou [2, 3].

For the symmetric case, that is, $p=q=1 / 2$, we have the following interesting corollary which may be justified by relation (3.10).

COROLLARY 4.2. For $p=q=1 / 2$, the $\operatorname{pmf} P(n), n \geq 0$, of the random variable $W$ is expressed in terms of the numbers $A_{n}^{(k, r)}$ as

$$
P(n)=\frac{A_{n-k}^{(k, r)}+A_{n-r}^{(r, k)}}{2^{n}} .
$$

Next, we turn our attention to some probability problems related with lengths of success and failure runs. Let $L_{n}^{(1)}, L_{n}^{(0)}$, and $X_{n}$ be random variables denoting the length 
of the longest success run, the length of the longest failure run, and the outcome of the $n$th trial, respectively, in $n$ independent Bernoulli trials with common success probability $p(0<p<1)$. Then we have the following theorem.

THEOREM 4.3. Let $A_{n}^{(k, r)}(x)$ be the sequence of the extended Fibonacci polynomials. Then, for $n \geq 1$,

$$
\operatorname{Pr}\left(L_{n}^{(1)}<k, L_{n}^{(0)}<r\right)=p^{n} A_{n}^{(k, r)}\left(\frac{q}{p}\right)+q^{n} A_{n}^{(r, k)}\left(\frac{p}{q}\right) .
$$

Proof. Noting that

$$
P_{1}(n+k)=p^{k} \operatorname{Pr}\left(L_{n}^{(1)}<k, L_{n}^{(0)}<r, X_{n}=0\right), \quad n \geq 1,
$$

it follows from relation (4.6) that

$$
\operatorname{Pr}\left(L_{n}^{(1)}<k, L_{n}^{(0)}<r, X_{n}=0\right)=p^{n} A_{n}^{(k, r)}\left(\frac{q}{p}\right), \quad n \geq 1 .
$$

In a similar way, using relation (4.7), we may obtain that

$$
\operatorname{Pr}\left(L_{n}^{(1)}<k, L_{n}^{(0)}<r, X_{n}=1\right)=q^{n} A_{n}^{(r, k)}\left(\frac{p}{q}\right), \quad n \geq 1 .
$$

The obvious identity

$$
\operatorname{Pr}\left(L_{n}^{(1)}<k, L_{n}^{(0)}<r\right)=\sum_{i=0}^{1} \operatorname{Pr}\left(L_{n}^{(1)}<k, L_{n}^{(0)}<r, X_{n}=i\right),
$$

along with relations (4.11) and (4.12), completes the proof of Theorem 4.3.

An alternative formula of the probability $\operatorname{Pr}\left(L_{n}^{(1)}<k, L_{n}^{(0)}<r\right)$, which may be easily justified by Theorem 4.3 and Proposition 3.5, is provided in the following corollary.

COROLLARY 4.4. Let $A_{n}^{(k, r)}(x)$ be the sequence of the extended Fibonacci polynomials. Then, for $n \geq 1$,

$$
\operatorname{Pr}\left(L_{n}^{(1)}<k, L_{n}^{(0)}<r\right)=\frac{p^{n+1}}{q} \sum_{i=0}^{k-1} \frac{q}{p} A_{n-i}^{(k, r)}\left(\frac{q}{p}\right) .
$$

We note that the evaluation of the probabilities $\operatorname{Pr}\left(L_{n}^{(1)}<k, L_{n}^{(0)}<r\right)$ through Theorem 4.3 (or Corollary 4.4) and Proposition 3.4 seems to be more efficient compared with a respective result of Antzoulakos and Philippou [3].

In the following corollary we give alternative derivations of (1.4) and (1.5).

COROLLARY 4.5. Let $F_{n}^{(k)}(x)$ be the sequence of Fibonacci-type polynomials of order $k$. Then, for $n \geq 1$,

$$
\begin{gathered}
\operatorname{Pr}\left(L_{n}^{(1)}<k\right)=\frac{p^{n+1}}{q} F_{n+2}^{(k)}\left(\frac{q}{p}\right), \\
\operatorname{Pr}\left(N_{k}=n+k\right)=p^{n+k} F_{n+1}^{(k)}\left(\frac{q}{p}\right) .
\end{gathered}
$$


Proof. First we observe that

$$
\lim _{r \rightarrow \infty} \operatorname{Pr}\left(L_{n}^{(1)}<k, L_{n}^{(0)}<r\right)=\operatorname{Pr}\left(L_{n}^{(1)}<k\right) .
$$

Corollary 4.4 and relation (3.12) imply that

$$
\lim _{r \rightarrow \infty} \operatorname{Pr}\left(L_{n}^{(1)}<k, L_{n}^{(0)}<r\right)=\frac{p^{n+1}}{q} \sum_{i=0}^{k-1} \frac{q}{p} F_{n+1-i}^{(k)}\left(\frac{q}{p}\right) .
$$

The proof of relation (4.15) then follows by relations (1.1), (4.17), and (4.18).

Next, letting $r \rightarrow \infty$, relations (4.11) and (3.12) yield

$$
\operatorname{Pr}\left(L_{n}^{(1)}<k, X_{n}=0\right)=p^{n} F_{n+1}^{(k)}\left(\frac{q}{p}\right), \quad n \geq 1 .
$$

Since $\operatorname{Pr}\left(N_{k}=n+k\right)$ can be written in the form

$$
\operatorname{Pr}\left(N_{k}=n+k\right)=p^{k} \operatorname{Pr}\left(L_{n}^{(1)}<k, X_{n}=0\right), \quad n \geq 1,
$$

we readily obtain relation (4.16).

In ending, we note that using Theorems 4.3 and relation (4.15) we may derive several interesting results concerning probabilities which involve shortest or longest success and/or failure runs. For instance, letting $n \geq 1$, we have that

$$
\begin{aligned}
\operatorname{Pr}\left(L_{n}^{(1)}<k, L_{n}^{(0)} \geq r\right) & =\operatorname{Pr}\left(L_{n}^{(1)}<k\right)-\operatorname{Pr}\left(L_{n}^{(1)}<k, L_{n}^{(0)}<r\right) \\
& =\frac{p^{n+1}}{q} F_{n+2}^{(k)}\left(\frac{q}{p}\right)-p^{n} A_{n}^{(k, r)}\left(\frac{q}{p}\right)-q^{n} A_{n}^{(r, k)}\left(\frac{p}{q}\right) .
\end{aligned}
$$

\section{REFERENCES}

[1] S. Aki, N. Balakrishnan, and S. G. Mohanty, Sooner and later waiting time problems for success and failure runs in higher order Markov dependent trials, Ann. Inst. Statist. Math. 48 (1996), no. 4, 773-787.

[2] D. L. Antzoulakos and A. N. Philippou, Derivation of the probability distribution functions for succession quota random variables, Ann. Inst. Statist. Math. 48 (1996), no. 3, 551-561.

[3] _ Probability distribution functions of succession quotas in the case of Markov dependent trials, Ann. Inst. Statist. Math. 49 (1997), no. 3, 531-539.

[4] N. Balakrishnan and M. V. Koutras, Runs and Scans with Applications, Wiley Series in Probability and Statistics, John Wiley \& Sons, New York, 2002.

[5] M. Ebneshahrashoob and M. Sobel, Sooner and later waiting time problems for Bernoulli trials: frequency and run quotas, Statist. Probab. Lett. 9 (1990), no. 1, 5-11.

[6] W. Feller, An Introduction to Probability Theory and Its Applications. Vol. I, 3rd ed., John Wiley \& Sons, New York, 1968.

[7] M. V. Koutras, Consecutive-k, r-out-of-n:DFM systems, Microelectron. Reliab. 37 (1997), no. 4, 597-603.

[8] A. N. Philippou, C. Georghiou, and G. N. Philippou, Fibonacci polynomials of order k, multinomial expansions and probability, Int. J. Math. Math. Sci. 6 (1983), no. 3, 545-550.

[9] _ A generalized geometric distribution and some of its properties, Statist. Probab. Lett. 1 (1983), no. 4, 171-175. 
[10] __ Fibonacci-type polynomials of order $k$ with probability applications, Fibonacci Quart. 23 (1985), no. 2, 100-105.

[11] A. N. Philippou and F. S. Makri, Longest success runs and Fibonacci-type polynomials, Fibonacci Quart. 23 (1985), no. 4, 338-346.

[12] A. N. Philippou and A. A. Muwafi, Waiting for the K th consecutive success and the Fibonacci sequence of order K, Fibonacci Quart. 20 (1982), no. 1, 28-32.

Demetrios L. Antzoulakos: Department of Statistics and Insurance Science, University of Piraeus, 80 Karaoli \& Dimitriou street, 18534 Piraeus, Greece

E-mail address: dantz@unipi.gr 


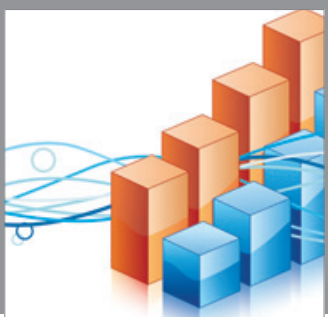

Advances in

Operations Research

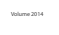

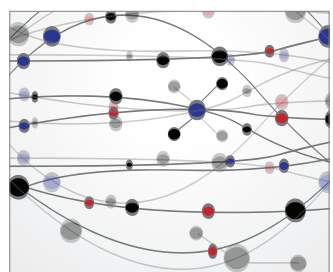

\section{The Scientific} World Journal
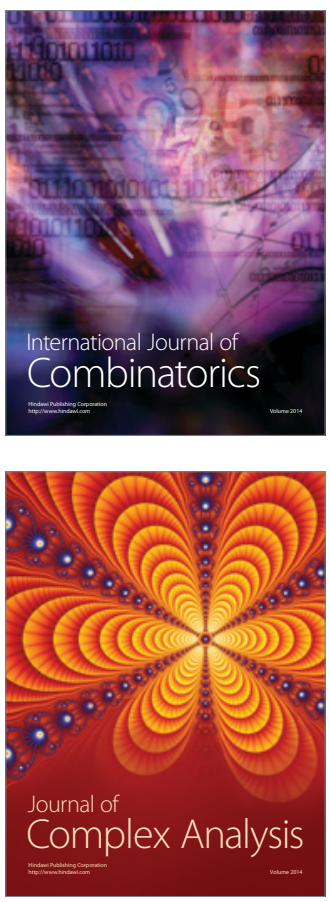

International Journal of

Mathematics and

Mathematical

Sciences
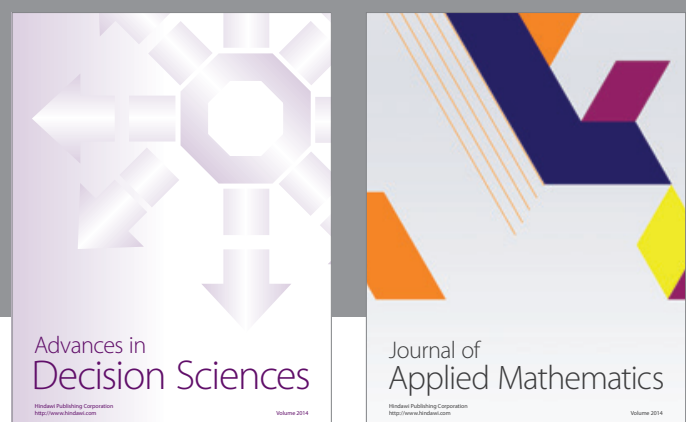

Journal of

Applied Mathematics
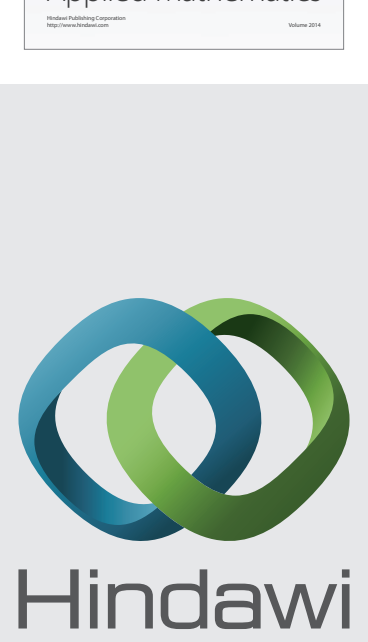

Submit your manuscripts at http://www.hindawi.com
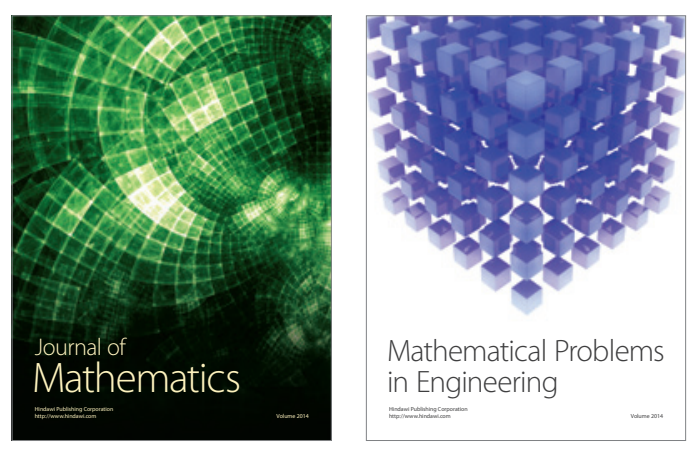

Mathematical Problems in Engineering
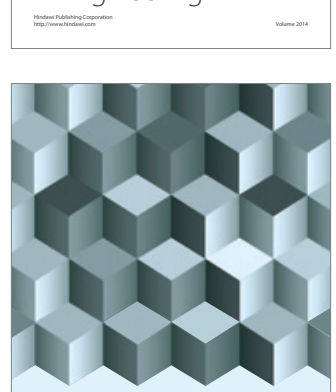

Journal of

Function Spaces
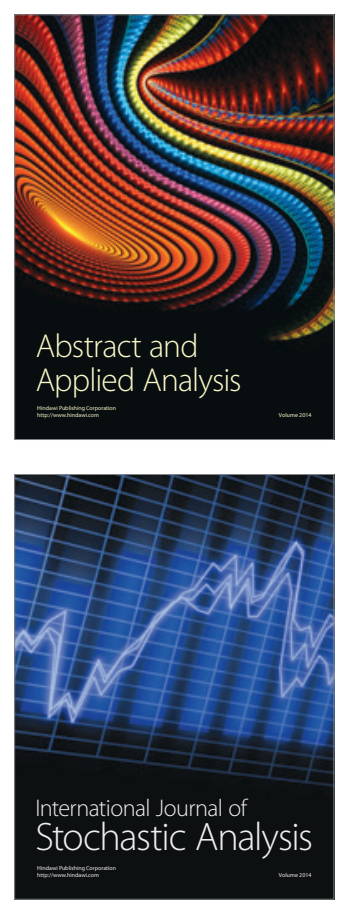

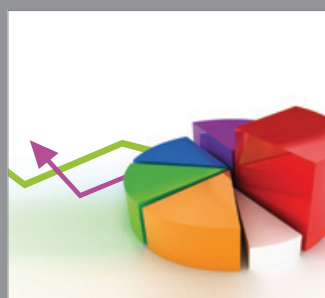

ournal of

Probability and Statistics

Promensencen
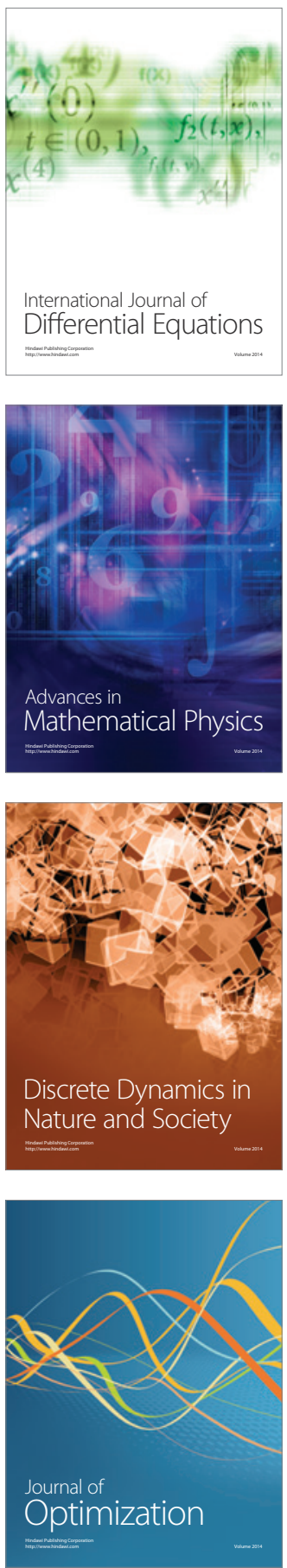